\title{
Et si Mélusine et Geoffroy la Grande Dent étaient des géants?
}

\section{Myriam White-Le Goff}

\section{(2) OpenEdition \\ 1 Journals}

Édition électronique

URL : https://journals.openedition.org/crm/778

DOI : $10.4000 / \mathrm{crm} .778$

ISSN : 1955-2424

Éditeur

Honoré Champion

\section{Édition imprimée}

Date de publication : 30 décembre 2006

Pagination : 305-313

ISSN : 1272-9752

\section{Référence électronique}

Myriam White-Le Goff, «Et si Mélusine et Geoffroy la Grande Dent étaient des géants ? », Cahiers de recherches médiévales [En ligne], 13 | 2006, mis en ligne le 30 novembre 2009, consulté le 15 décembre 2022. URL : http://journals.openedition.org/crm/778 ; DOI : https://doi.org/10.4000/crm.778 


\section{rM}

\section{Et si Mélusine et Geoffroy la Grande Dent étaient des géants?}

Les figures de géants ne manquent pas dans la littérature médiévale. Certaines ont été évoquées lors d'un colloque qui s'est tenu à l'université d'Artois, à Arras, les 24 et 25 novembre 2005. En entendant différentes communications, je n'ai pu m'empêcher de penser à la légende de Mélusine, à la fée et à son fils chéri, Geoffroy la Grande Dent. En effet, certains ont déjà envisagé Mélusine comme une géante et il m'a semblé qu'on pouvait aller plus loin encore, en interrogeant également la figure de Geoffroy. J'ai choisi de m'intéresser aux personnages de la légende telle que nous la rapporte Jean d'Arras en 1392. On connaît l'histoire de la fée poitevine qu'épouse Raymondin. Geoffroy la Grande Dent est son sixième fils, né après huit ans de mariage. Il «apporta sur terre une dent qui lui yssoit hors de la bouche plus d'un pouce ${ }^{1}$. Je m'attacherai au personnage littéraire, plutôt qu'à cet historique Geoffroy de Lusignan qui semble avoir incendié l'abbaye de Maillezais en 1232. Paradoxalement, alors que j'aurais pu trouver mille vrais géants, mille personnages appelés géants, j'ai choisi de m'intéresser à une dame qu'on ne qualifie que très timidement de fée et à Geoffroy, ce protagoniste qui n'est pas un géant, même s'il est «grans, haulx et fourniz et fort a merveilles, hardiz et crueulx. » et que «chascun le doubt[e] qui en ouoit parler $»^{2}$. Nulle part dans l'œuvre de Jean d'Arras, Geoffroy n'est présenté comme un géant, tout au plus comme un «diable»! Et pourtant... Certes, il ne faut pas confondre géant et grand chevalier ${ }^{3}$. Toutefois, il m'apparaît qu'il peut être fructueux de questionner un moment les créaturescréations étranges que sont Mélusine et Geoffroy, en lien avec une problématique 'gigantale', depuis la fée civilisatrice mais sauvage jusqu'au grand fils impétueux bien que sage, au cœur de tous les secrets familiaux.

Dès le début du roman de Jean d'Arras, la nature de Mélusine est mise en question. Elle est indécise et l'auteur ne souhaite jamais vraiment la définir. Il évoque seulement des créatures «lesquelles aucuns appeloient luitons, aucuns autres les faes, aucuns autres bonnes dames qui vont de nuit ${ }^{4}$. L'essence de Mélusine est profondément mystérieuse, multiple et masquée par les métamorphoses et les fauxsemblants. La belle dame dont Raymondin est épris ressemble pourtant à une espèce de géante bâtisseuse : elle « fist bastir la ville de Lusignen et fonder les murs sur la vive roche. Et la fit estoffer de fortes tours drues, machicolees et a terrace, et les murs machicoléz et alees au couvert dedens la muraille ${ }^{5}$. Jean d'Arras rappelle

\footnotetext{
${ }^{1}$ Jean d'Arras, Mélusine ou La Noble Histoire de Lusignan, nouvelle éd. de J.-J. Vincensini, d'après le ms. de l'Arsenal, Le Livre de Poche, coll. «Lettres gothiques », Paris, 2003, p. 294. ${ }^{2}$ Ibidem, p. 294.

${ }^{3}$ Voir à ce sujet, Francis Dubost, Aspects fantastiques de la littérature narrative médiévale : XII ${ }^{e}$-XIII ${ }^{e}$ siècles : l'autre, l'ailleurs, l'autrefois, Paris, Champion, Nouvelle bibliothèque du Moyen Âge, 15, 1991, chapitre 19, «Le fantastique du monstre, les géants », p. 568 sq.

${ }^{4}$ Ibid., p. 116.

${ }^{5}$ Ibid., p. 262.
}

Cahiers de Recherches Médiévales (XII -XVe s.), 13, 2006 
également que Mélusine est d'abord défricheuse : «apperceurent sur la roche de la Fontaine de Soif grans trencheiz et arbres abbatus d'une part et d'autre dont ilz se prindrent fort a merveiller, car oncques mais ilz n'avoient veu trencheiz. Remondin, qui bien s'apperceut que sa dame avoit ouvré, se teust $»^{6}$. En outre, elle inspire à son époux Raymondin un stratagème magique pour délimiter son territoire, qui n'est pas sans évoquer une gigantisation de la matière. En effet, le jeune homme réclame qu'on lui fasse don d'autant de terre qu'il en pourra enceindre avec une peau de cerf'. Or cette peau parait presque surnaturelle puisqu'elle permet de circonscrire un très vaste territoire, qui «comprenoit bien deux lieues de tour ${ }^{8}$, sous le regard effaré des assistants! Le processus est expliqué par la confection, avec la peau, d'une fine lanière formant un écheveau. Or cet agrandissement démesuré de la surface que peut enclore une peau de cerf est associé à un autre motif qui peut faire soupçonner une appartenance au monde gigantal: la peau commence significativement par enclore une montagne, habitat-vestige de géants, s'il en est'. Jean d'Arras affirme encore que les édifices qu'érigent Mélusine par féerie sont d'une taille jusqu'alors inconnue et surtout qu'ils se construisent à une vitesse prodigieuse, inhumaine : «Moult fu la forteresce grant et fort a merveilles ! Et sachiéz que le conte de Poictiers et tuit ly nobles et les menuz peuples du paÿs furent tous esbahiz comment si grant ouvraige pouoit estre en si pou de temps faiz ne achevéz $»^{10}$. L'évocation des nombreux maçons convoqués par la fée pour réaliser ses projets et originaires d'on ne sait trop où ne serait alors que la preuve d'une volonté de rationalisation du mythe. On pourrait y voir, à la façon de J. Le Goff ${ }^{11}$, la volonté touchante de Mélusine de vivre parmi les hommes. Il n'en demeure pas moins, qu'elle laisse dans le monde des vestiges de pierres, comme les géants de légendes qu'on devine sous les ruines d'édifices extraordinaires.

En contrepoint avec cette Mélusine civilisatrice et fondatrice, la fée se transforme en une ogresse relativement effrayante quand, au moment de quitter le monde des hommes, elle dicte la mise à mort de son propre fils, Horrible, qu'il faudra surprendre par la ruse et étouffer... Elle explique ce choix par le danger que sa progéniture ferait courir au monde humain, mais la décision de la mère n'en est pas moins vecteur d'inquiétude. On comprend l'interrogation non seulement chtonienne mais aussi mythique ou tellurique sous-jacente à l'histoire de cette Mélusine primordiale, amante, mère et défricheuse, quand on se souvient que c'est aussi Mélusine, la fille aînée de Pressine, qui est l'instigatrice, à l'origine de la

\footnotetext{
${ }^{6}$ Ibid., p. 184.

${ }^{7}$ Cf. la ruse de Didon qui demande autant de terre que pourrait comprendre un peau de taureau puis découpe le cuir en petites courroies très fines qui lui permettent de délimiter suffisamment de terre pour y fonder la cité de Carthage, Éneas v. 391sq. qui suit l'Énéide, I, v. 368. De manière générale, différents parallèles existent entre Didon et Mélusine, toutes deux fondatrices, constructrices et maitresses de leur domaine. Les deux femmes sont également mal aimées par un homme qui les trahit.

${ }^{8}$ Ibid., p. 184.

${ }^{9}$ Voir le passage, ibid., p. 184.

${ }^{10}$ Ibid., p. 214.

11 «Mélusine maternelle et défricheuse", Annales E.S.C., 1971, p. 587-603, repr. dans Pour un autre Moyen Âge, Paris, Gallimard, Bibliothèque des Histoires, 1977, p. 307-331.
} 
décision de punir la trahison de son père ${ }^{12}$, en l'enfermant dans la montagne magique de Brumborenlion. Elle propose avec assurance à ses deux sœurs, Mélior et Palestine: «j'ay advisié, se il vous semble bon, que nous l'enclouons en la merveilleuse montaigne de Norhonbelande nommee Brumbloremlion, et de la n'ystra de toute sa vie $»^{13}$. N'est-ce pas là un châtiment que seule une fille géante, élevée par sa mère dans les montagnes ${ }^{14}$ de l'île Perdue d'Avalon, pouvait imaginer? L'assimilation de Mélusine à une géante est particulièrement lisible dans l'iconographie où la fée se métamorphose en un être anguipède qui ressemble étrangement aux représentations des géants de la tradition grecque. Rabelais constatait de même la nature «adouillique » de la fée qui la rapprochait des géants ${ }^{15}$. Certains n'hésitent d'ailleurs pas à voir en Mélusine la vraie mère de Gargantua, dont Gargamelle ne serait qu'un masque, ou encore à faire de Geoffroy une image affaiblie du fameux géant rabelaisien ${ }^{16}$. Du même point de vue iconographique, Mélusine est encore parfois représentée sous la forme d'un dragon, notamment lors de son envol ${ }^{17}$. Or on associe également le dragon au géant. Est-ce à dire qu'on assimile indirectement Mélusine à une géante ? Quoi qu'il en soit, en conséquence des caractéristiques 'gigantales' de leur mère, les fils de Mélusine ont parfois été perçus comme des géants. Jean Markale affirme catégoriquement: «les fils de Mélusine ont une taille et des allures peu communes qui les apparentent à des personnages gigantesques. En réalité, ce sont des géants, non seulement par leurs particularités, mais par leur comportement $»^{18}$. Il montre que les fils de la fée sont des «géants rationalisés»" ${ }^{19}$. Geoffroy semble être l'héritier direct du gigantisme maternel. Dans le registre de l'anecdote, un médecin, le Docteur Marcel Baudouin, s'est intéressé en 1910 à la pathologie du fils de Mélusine, dans un article intitulé «quelle était la grande dent de Geoffroy-la-Grande-Dent?» Il observe qu'on nomme

\footnotetext{
${ }^{12}$ Elinas a enfreint la promesse faite à son épouse de ne pas la voir en couches, sur les instances séductrices et malintentionnées de son fils jaloux, Mataquas.

${ }^{13}$ Jean d'Arras, op.cit., p. 132-4.

${ }^{14}$ Jean d'Arras rapport que, trahie par son époux, Pressine a dû le quitter avec ses trois filles et qu'elle les a élevées en Avalon, les menant contempler l'Écosse tous les matins, du sommet d'une haute montagne que l'histoire appelle Eleneos : « une haulte montaigne laquelle estoit appelle, si comme l'ystoire dit, Bleneos qui vault autant dire en françois comme «montaigne florie »» (ibid., p. 132).

${ }^{15}$ Quart livre, chapitre 38, Pantagruel, chapitre V.

${ }^{16}$ G.-E. Pillard, Le Vrai Gargantua: mythologie d'un géant, Paris, Imago, 1987, p. 185. Mélusine fait ici partie de la mythologie française du géant. Voir également J. Markale, Mélusine, Paris, Albin Michel, 1993, rééd. de Mélusine et l'Androgyne, Retz, Paris, 1983, p. 69. Ou encore F. Clier-Colombani, op. cit., p. 96 : «Mélusine fait partie des géants qui peuplent la France médiévale et son fils Geoffroy la Grand'dent, comme elle, appartient à la même famille que Pantagruel et Gargantua ».

${ }^{17}$ Peut-être que cette représentation est inspirée par l'histoire de l'épouse de Henno aux Grandes Dents chez Gautier Map (De Nugis curialium, les Contes des gens de cour) qui se transforme en dragon car elle ne supporte par le contacte de l'hostie et de l'eau bénite.

${ }^{18}$ J. Markale, op. cit., p. 69. Les fils de Mélusine seraient des géants car ils seraient nés des amours d'un mortel et d'une déesse ou du moins d'un être surnaturel. J. Markale rapproche leur cas de celui d'Hercule ou des géants bibliques de Genèse VI, 1-4.

${ }_{19}$ J. Markale, op. cit., p. 71. L'espace que la seule raison permet d'ouvrir serait donc bien étroit...
} 
les anomalies dentaires de cette nature «géantisme $»^{20}$. Souvenir de la nature de géant de Geoffroy? Cela est bien possible, surtout si l'on se rappelle que quand on trouve des vestiges de la présence hypothétique de géants sur terre, il s'agit très souvent de très grandes dents, précisément ${ }^{21} \ldots$

Geoffroy tient résolument une place à part entre les fils de la fée. En effet, avant toute chose, et, il est vrai, comme beaucoup de preux chevaliers, il se caractérise par une très grande taille qui ne sera surpassée que par celle de son frère cadet Horrible. La taille exceptionnelle des fils de Mélusine semble suggérer que la merveille que leur confère leur ascendance maternelle peut difficilement être contenue par l'humaine nature et tente de dépasser les limites qu'elle lui impose. La part féerique des fils déborde la norme de notre monde. Qui plus est, entre ces fils, c'est surtout Geoffroy que Jean d'Arras invite à considérer comme une force de la nature. Dans le roman, il est le vainqueur de plusieurs géants ${ }^{22}$. Il parvient à dominer Gardon et Grimaut. Le géant Gardon habitait sur la montagne de la Tour de Monjouet. Alors qu'il ravageait les terres de Raymondin, de Guérande jusqu'à La Rochelle, «nouvelles en vindrent a Remond, qui moult en fut doulent; mais il n'en monstra nul samblant afin que Gieffroy ne le sceust, pour doubte qu'il n'alast combatre le jayant; car il le sentoit de si grant cuer qu'il ne lairoit point qu'il n'y alast $»^{23}$. Geoffroy réveille effectivement le géant assoupi et le provoque en duel. Il lui coupe un bras, une jambe et la tête, qu'il fait envoyer à son père ${ }^{24}$. Le géant Grimaut du pays de Northumberland, quant à lui, habitait près de la montagne de Brumbeloy, et opprimait les habitants. Ceux-ci ont demandé l'aide de Geoffroy dont la réputation leur était parvenue. Le fils de la fée a volontiers accepté et s'en est pris à l'horrible tortionnaire. Après un dernier combat, le géant a disparu dans un pertuis de la montagne. Mais le héros a retrouvé Grimaut et l'a tué. Ces combats contre les géants ont souvent été retenus par les illustrateurs de la légende car ils participaient à la valorisation morale de Geoffroy et renforçaient le caractère spectaculaire de la narration $^{25}$. Personne avant Geoffroy n'avait pu venir à bout de ces créatures gigantesques. En cela, Geoffroy rejoint la tradition des chevaliers vainqueurs de géants. Le Roman de Brut rapporte que le roi Arthur lui-même s'est distingué par un tel exploit! Les frères de Geoffroy sont également d'excellents chevaliers, mais il semble être le seul de la famille à avoir la force nécessaire pour parvenir à accomplir cette tâche spécifique et on pourrait penser qu'il réussit, seul, à se mesurer aux géants car il est, dans une certaine mesure, le seul fils de la fée à être leur égal.

\footnotetext{
${ }^{20}$ Poitiers, impr. de Blais et Roy, 1910, p. 6.

${ }^{21}$ Saint Augustin, par exemple, déclare avoir trouvé sur une plage une molaire gigantesque (Questions sur la Genèse, I, 3).

${ }^{22}$ Dans l'introduction de son édition de Mélusine, J.-J. Vincensini y voit une partie de l'œuvre civilisatrice de Geoffroy (ibid., p. 35-6). Pour lui, « Geoffroy, héritier de Raymondin, occupe la place de l'éradicateur individuel des derniers vestiges de l'ordre ancien, purificateur des maléfiques représentants d'une anti-culture primitive » (p. 37).

${ }^{23}$ Ibid., p. 654.

${ }^{24}$ La mort du géant par décapitation, n'est pas sans évoquer celles de Goliath ou surtout d'Holopherne.

${ }_{25}$ Voir à ce sujet, F. Clier-Colombani, Images de Mélusine à la fin du moyen-âge, Lille 3, ANRT, 1988.
} 
Jean d'Arras souligne en outre la singularité voire la supériorité de Geoffroy en montrant qu'il est également celui qui se porte au secours de ses frères et qu'il est considéré par leurs ennemis comme le plus redoutable de la fratrie. On pense, par exemple, à la réaction de l'interprète, quand le sultan de Damas lui annonce qu'il devra s'adresser à Geoffroy: «Moy, dist le druceman, a l'eure et au jour que je l'approucheray, que je puisse, qu'il n'ait une grosse riviere ou les tours et les murs de Damas ou de quelque autre lieu fort, me puist Mahon confondre ! $»^{26}$. Le sultan lui-même affirme : «Par Mahon, (...) je croy que cellui au grant dent a le diable ou corps ! ${ }^{27}$. Ainsi, Geoffrey est le fils de Mélusine par excellence. La nature féerique de sa mère se retrouve partiellement en lui. Comme elle, également, il montre à Raymondin qu'il souhaite mater les parts excessives de sa nature: quant il tue Gardon, il fait porter la tête en tribu à son père, comme s'il voulait lui montrer qu'il renonçait à cette nature inquiétante que lui lègue sa mère, pour choisir d'être un grand chevalier humain. Si Geoffroy est géant, il ne semble pas l'être pleinement car le géant Gardon, quoique reconnaissant la vaillance et la stature exceptionnelle de son adversaire, se rit bien de voir un homme seul s'en prendre à lui ${ }^{28}$. De même, au moment d'entrer dans la montagne par le même pertuis qu'a emprunté le géant Grimaut, le chevalier Geoffroy reconnaît : «le jayant est plus grant et plus groz que ne suiz $»^{29}$.

Toutefois, d'autres aspects du roman de Jean d'Arras semblent aller dans le sens d'une singularisation de Geoffroy. On sait que le conte mélusinien implique une triste issue pour l'idylle entre un mortel et un être surnaturel ${ }^{30}$. Or c'est précisément Geoffroy qui a indirectement causé la rupture de ses parents, suivi du départ spectaculaire et définitif de Mélusine du monde des humains. La fée s'envole par la fenêtre du château familial à la suite des reproches qui lui adresse son époux Raymondin. De fait, Geoffroy a incendié l'abbaye dans laquelle son frère Fromont désirait entrer et a ainsi causé sa mort et celle des moines. Cet acte terrible cause la révolte et le désespoir de son père qui l'impute à Mélusine. Il lui reproche sa nature monstrueuse qui expliquerait le comportement diabolique de sa progéniture. Déjà, au plan de la narration, Jean d'Arras choisit le moment où Geoffroy part tuer son premier géant, Gardon, pour interrompre le récit de l'aventure du chevalier et raconter la première transgression par Raymondin de la promesse faite à Mélusine de ne pas chercher à la voir le samedi. Ainsi, c'est alors que Geoffroy est en quête de Gardon que, soupçonneux, Raymondin espionne son épouse le samedi et découvre le secret de sa métamorphose. Le couple extraordinaire ne tient déjà plus que par la repentance de Raymondin et le pardon implicite de Mélusine qui, bien qu'elle se soit aperçue de la trahison, ne fait mine de rien. Il semble bien que le combat qui met aux prises Geoffroy avec son double géant rappelle et renforce la conscience de l'incompatible amour de la fée, représentante d'une autre nature, et du

${ }^{26}$ Éd. cit., p. 618.

${ }^{27}$ Ibid., p. 626.

${ }^{28}$ Éd. cit., p. 670.

${ }^{29}$ Ibid., p. 716.

${ }^{30}$ Voir L. Harf-Lancner, Les Fées au Moyen Âge, Paris, Champion, Nouvelle Bibliothèque du Moyen Âge, 8, 1984 et Le Monde des fées dans l'occident médiéval, Paris, Hachette Littératures, 2003. 
mortel. Avec une grande cohérence, le désaveu public de Mélusine par Raymondin et ses conséquences précèdent directement le meurtre du second géant, Grimaut, par Geoffroy. La nature profonde de Mélusine et de son fils ressortent de toutes parts et font pressentir l'issue fatale. Il ne semble pas que ce soit par hasard que Geoffroy soit à l'origine de la séparation définitive du couple parental. En effet, quel autre enfant souligne mieux que lui la difficile conciliation des deux mondes et des deux natures, humains et féeriques, qu'incarnent les deux parents? Horrible ? Non. Seul Geoffroy ressemble à ce point à sa mère qu'il est affublé d'une grande dent proche de celle du sanglier ${ }^{31}$, avatar probable de la fée au début du roman, qui déclenche la rencontre entre Raymondin et sa future épouse. Geoffroy est le fils adoré, élu par sa mère, tandis qu'elle prémédite la mort d'Horrible ${ }^{32}$. Geoffroy, à la différence de ses frères Urien, Eudes, Guion, Renaud, Antoine, Fromont... garde pleinement ses deux natures et l'effort de Mélusine ne parvient pas à en faire un simple humain, un peu étrange, comme semblent l'être ses frères ${ }^{33}$.

Geoffroy est résolument hors du commun. Comme certains géants mythiques, il semble être le détenteur d'une sagesse profonde que ne masque seulement de manière superficielle une forme de sauvagerie ou de barbarie. En cela encore, il se rapproche de nombreuses autres figures de géants. Seule Mélusine, la fée, peut comprendre le bien-fondé de certains de ces actes, comme l'incendie de l'abbaye par exemple: "Gieffroy (...) a fait son oultraige par son courage merveilleux et fort, sachiéz que de certain c'est pour le pechié des moines, qui estoient de mauvaise vie et desordonnee. Et en a nostre Seigneur voulu avoir la punicion, combien que ceste chose soit incongnoissable quant a humaine creature $»^{34}$. Geoffrey aurait finalement fait preuve d'une certaine justice, en punissant des moines coupables de graves péchés ; il aurait presque agi sur instigation divine. Le géant serait une forme intermédiaire entre les hommes et Dieu, dernier représentant d'un état antérieur de la Création. C'est pourquoi, Mélusine recommande à Raymondin de garder Geoffroy auprès de lui «car il fera un tresvaillant homme $»^{35}$. De plus, c'est encore Geoffroy qui vengera ses parents en tuant le traître comte de Forestz qui avait, par malveillance, stimulé la jalousie de Raymondin et l'avait ainsi poussé à trahir sa promesse envers son épouse. C'est lui encore qui accompagnera son père dans sa foi, qui reconstruira l'abbaye qu'il a détruite, qui se confessera au pape, cherchera à accomplir le bien, et qui permettra le rachat total du domaine parental, en se mesurant au mystérieux personnage qui perçoit un tribut annuel sur le bien familial.

\footnotetext{
${ }^{31}$ Déjà, ce sanglier est considéré comme monstrueusement grand.

${ }^{32}$ C'est même là l'essentiel de son testament, au moment de quitter le monde des hommes. Horrible présenterait une trop grave menace pour l'humanité pour qu'on le laisse vivre.

${ }^{33}$ Rappelons que les fils de Mélusine sont tous stigmatisés par des tares physiques étonnantes (voir Jean d'Arras, op. cit., p. 294-6), sauf peut-être les deux derniers, Raimonnet et Thierry, qui semblaient attester de l'intégration totale de la fée au monde des hommes, même si cela n'était qu'illusoire. Dans son introduction à son édition du texte de Jean d'Arras, J.-J. Vincensini fait très justement remarquer que «sa mère dispense [Geoffroy] de tous conseils, moraux ou juridiques, quand il part outre-mer; sa terrible force suffit» (p. 16) ; alors qu'elle fait d'abondantes recommandations à ses autres fils.

${ }^{34}$ Ibid., p. 692.

${ }^{35}$ Ibid., p. 698.
} 
Ce dernier passage du roman est particulièrement étonnant et merveilleux. Geoffroy y est seul capable de combattre un chevalier faé ${ }^{36}$. Par son ascendance maternelle, il se mesure à l'altérité féerique sans grande difficulté. Son adversaire, celui qui reçoit l'argent consenti par les intendants du domaine, demeure longtemps non-identifié puis on évoque un grand chevalier à la voix impressionnante qui, là encore, n'est pas sans évoquer une figure de géant, notamment dans le fait qu'il peut tenir en sa main le sommet d'une tour: "tous les ans, le derrenier jour d'aoust, venoit une grant main et prenoit le pommel de la Tour Poictevine, et l'en esrachoit si fort qu'il abatoit grant partie de la couverture de la tour $»^{37}$. D'ailleurs la façon dont Geoffroy aborde ce personnage est une forme de redoublement de la manière dont il s'adresse au premier géant qu'il combat, Gardon, auquel il dit : «Filz a putain, viens a moy! Je t'apporte l'argent des patiz que les gens monseigneur mon pere te doivent $»^{38}$. La rencontre avec cet être extraordinaire est une forme de nouvelle confrontation avec un géant.

De plus, c'est également à Geoffroy que revient le privilège d'accomplir un autre acte extraordinaire : la découverte du tombeau d'Elinas, le père de Mélusine ${ }^{39}$. Le fils de la fée accède ainsi à sa totale identité et il donne l'une des clés essentielles du lignage des Lusignan. Cela est fondamental pour le projet de Jean d'Arras qui annonce dès le début de son roman : «et me orréz declairer la noble lignie qui en est yssue qui regnera jusques en la fin du monde, selon ce qu'il appert qu'elle a regné jusqu'à ore $»^{40}$. Geoffroy révèle ensuite à son père la véritable nature et l'histoire de sa bien-aimée Mélusine. Il a ainsi un rôle particulier dans l'œuvre car c'est lui qui en dévoile la signification et qui découvre le mystère. Par là, il est particulièrement valorisé. Qui plus est, c'est en suivant le géant Grimaut, qui tente de lui échapper en entrant dans un pertuis, que Geoffroy découvre ce secret des origines, comme s'il fallait nécessairement en passer par la figure gigantale et par son expression naturelle, la montagne, pour accéder à soi, quand on est un géant, ne serait-ce que partiellement. Le sort de Grimaut et celui de Geoffroy sont profondément liés, comme en attestent «les saiges astronomiens» qui «ont dit que le jayant ne puet mourir fors par ${ }^{41}$ le fils de Mélusine. Suivre Grimaut dans les entrailles de la terre est une épreuve initiatique, une véritable descente aux enfers. Grimaut n'est d'ailleurs que l'un des nombreux géants auxquels la grand-mère de Geoffroy, Pressine, a confié la garde du tombeau de son époux Elinas : «et estably un jayant fier et horrible qui gardoit ce lieu et tenoit tout le païs en subjection et pactis. Et ainsi le tindrent pluseurs jayans après lui jusqu'a la venue Gieffroy au grant dent ${ }^{42}$. Il est d'ailleurs significatif que la mère de Mélusine soit en mesure de commander aux géants ! Cela laisse présager de sa propre nature...

Ainsi, si Mélusine peut être considérée comme une géante, certains voient également en elle une figuration de déesse-mère. Jean Markale affirme qu'elle est

\footnotetext{
${ }^{36}$ Cet épisode n'est pas évoqué par Coudrette.

${ }^{37}$ Jean d'Arras, op. cit., p. 794.

${ }^{38}$ Ibid., p. 670.

${ }^{39}$ Ibid., p. 716 sq.

${ }^{40}$ Ibid., p. 118.

${ }^{41}$ Ibid., p. 710.

${ }^{42}$ Ibid., p. 138.
} 
«plus que jamais une Déesse-Mère des temps primordiaux, dont l'image s'est chargée d'éléments appartenant en grande partie à la tradition celtique. Mélusine est un des aspects, celtique et nord-occidental, de l'Être primordial qui a donné naissance à la race humaine et à la race des dieux $\aleph^{43}$. De fait, Jean d'Arras insiste abondamment sur la nature maternelle de la fée qui, même après avoir quitté le monde des hommes, revient en cachette allaiter ses deux derniers fils ${ }^{44}$. Or si Mélusine est une déesse, ses fils sont des enfants-dieux. D'ailleurs, le meurtre de Fromont par Geoffroy n'est pas sans évoquer un autre meurtre primordial, celui d'Abel par Caïn. Les enfants-dieux de Mélusine pourraient donc être à l'origine d'un nouvel ordre du monde. Pour G.-E. Pillard, "avec Geoffroy, un monde nouveau s'entrevoit: le monde des Géants, et aussi le monde des Héros. Ce fils est d'une autre lignée que celle de ses frères ${ }^{45}$. Sans aller aussi loin, on peut constater que Geoffroy se distingue encore de ses frères par le fait qu'il ne se marie pas. Ses aînés font de dignes mariages et fondent un important lignage: Urien épouse Hermine, la fille du roi de Chypre, Guion la pucelle Florie et devient roi d'Arménie, Antoine Christine, duchesse de Luxembourg, Renaud Aiglentine, fille du roi de Bohême. On sait combien l'écriture du roman de Jean d'Arras est liée à la célébration de certaines familles et à l'affirmation de la valeur de leurs ancêtres légendaires. Il écrit en 1392 pour deux descendants de la famille des Lusignan : Jean de Berry et sa sœur, Marie, duchesse de $\mathrm{Bar}^{46}$. Il peut donc sembler surprenant de donner une telle importance à un personnage qui n'aura justement pas de descendance. En réalité, Geoffroy est utile au projet. Avec lui, le lignage des Lusignan se débarrasse en quelque sorte de sa part féerique, quelque peu gênante. Ce fils qui était le porteur de l'ascendance surnaturelle, double de Mélusine, ne survivra que dans l' «hagiographie » fantasmatique de la famille mais il ne troublera pas de son sang merveilleux celui des Lusignan ${ }^{47}$. La race des géants qui perdure encore en Geoffroy doit s'éteindre en cette fin du Moyen Âge. Il est l'un de ses derniers représentants.

De manière générale, le roman de Mélusine est particulièrement intéressant en ce qui concerne la problématique qui nous intéresse car il défie l'axiologie traditionnelle du Moyen Âge qui fait souvent du géant un être maléfique ou diabolique. Ainsi, le grand chevalier faé dont Geoffroy est vainqueur est certes redoutable et rançonne les Lusignan, mais il n'est en rien diabolique puisqu'il demande la fondation d'une abbaye pour faire cesser sa persécution. Il semble être du côté de Dieu et de la religion, aussi affirme-t-il: «de par Dieu suiz je voirement $»^{48}$. L'argent qu'il perçoit ne paraît servir que de hauts et saints objectifs. Il permet d'ailleurs pleinement à Geoffroy, à cette occasion, de racheter le crime qu'il a commis en détruisant l'abbaye quelques années plus tôt. Sa rencontre est donc à la fois l'occasion de démontrer encore la prouesse du fils de Mélusine, mais

\footnotetext{
${ }^{43}$ J. Markale, op. cit., p. 72.

${ }^{44}$ Éd. cit., p. 708.

${ }^{45}$ G.-E. Pillard, op. cit., p. 86.

${ }^{46}$ Entre 1401 et 1405 , en réécrivant l'histoire de Mélusine, Coudrette veut renforcer les liens entre la famille des Parthenay et les Lusignan.

${ }^{47}$ Il faut ici bien faire la différence entre le personnage du roman et un Geoffroy la Grande Dent historique.

${ }^{48}$ Éd. cit., p. $788-90$.
} 
aussi de valoriser tout à fait son personnage au plan moral. Curieusement, ce perfectionnement du personnage de Geoffroy passe par une figure quasi sinon pleinement 'gigantale'... C'est en lien avec des figures de géants qu'apparaît la vraie nature de Geoffroy. Sa qualité de tueur de géants monstrueux est celle qui rattrape tous ses défauts et qui assure en partie le salut de son âme. Les figures de géants permettent indirectement la rédemption du héros. En s'élevant contre les mauvais géants, il refoule symboliquement une partie de sa propre nature, pour ne garder que le meilleur de lui-même. De même, l'ambivalence morale profonde des figures de géants se retrouve à propos de l'histoire de la sœur de Mélusine, Palestine. Elle est punie par sa mère pour avoir participé au châtiment de son père et est en conséquence enfermée à son tour dans la montagne du Canigou, avec le trésor de son père. Elle y est protégée par d'horribles monstres et survit dans l'espoir qu'un chevalier de son lignage viendra un jour la délivrer et utiliser son trésor pour conquérir la Terre promise. Paradoxalement, ces monstres, qui semblent également être des géants, deviennent des anges gardiens, protecteurs pour Palestine. On peut nuancer cette observation en montrant que ce relatif renversement moral du géant terrifiant devenu bon gardien se produit dans le contexte biaisé de la malédiction initiale pesant sur la captive. Dans la légende de Mélusine, il semble bien qu'on soit souvent confronté à un ordre du monde ancien qui bouleverse ou, du moins, interroge les frontières du bien et du mal... Les géants, comme Geoffroy quand il commet son impardonnable crime, seraient incompris, bien plus que mauvais. Ils appartiendraient à une réalité révolue, régie par des lois désormais incompréhensibles.

En somme, le personnage de Geoffroy à la Grande Dent, s'il n'est jamais explicitement considéré par Jean d'Arras comme un géant, mérite d'être envisagé comme bien plus qu'un grand chevalier. Comme Mélusine, il ne saurait être réduit à une humanité monstrueuse. Comme sa mère, il participe pleinement de la merveille. Je ne peux me résoudre à voir dans le réseau de rapprochements, de présomptions, de coïncidences qui apparaissent au fil du texte, l'expression d'un simple hasard ou la preuve d'une sur-interprétation forcenée, qui n'est d'ailleurs pas mon propos. Il ne s'agit pas de faire à toute force de Mélusine ou de Geoffroy des géants mais de montrer comment les personnages expriment la facilité et la liberté avec lesquelles les hommes du Moyen Âge pouvaient faire entrer dans leur représentation du monde des êtres hors-normes, cohabiter avec eux, leur confier des missions supérieures ou les inscrire dans leur lignage. Toutefois, cette sur-nature n'est pas seulement une bénédiction pour une famille ou un artifice romanesque de petite envergure. Elle permet de suggérer une altération des temps, de créer des personnages dont l'épaisseur et la singularité leur assurent une portée symbolique et littéraire hors du commun, qui expliqueront qu'on reprendra inlassablement la légende de la fée Mélusine et qu'on écrira, encore après le Moyen Âge, les aventures de Geoffroy la Grande Dent. D'ailleurs, dans le très récent album pour enfants «Geoffroy la Grand'Dent ", qui fait partie de la série Mélusine Fée serpente, le héros est présenté comme un géant ${ }^{49}$, comme s'il s'agissait d'une évidence.

Myriam White-Le Goff Université d'Artois

${ }^{49}$ S. Balland et D. Quella-Guyot, Geste éditions, 2001. 